\title{
INVARIANT SUBSPACES IN THE BIDISC AND WANDERING SUBSPACES
}

\author{
TAKAHIKO NAKAZI
}

(Received 26 September 2005; accepted 4 October 2006)

\author{
Communicated by A. L. Pryde
}

\begin{abstract}
Let $M$ be a forward-shift-invariant subspace and $N$ a backward-shift-invariant subspace in the Hardy space $H^{2}$ on the bidisc. We assume that $H^{2}=N \oplus M$. Using the wandering subspace of $M$ and $N$, we study the relations between $M$ and $N$. Moreover we study $M$ and $N$ using several natural operators defined by shift operators on $H^{2}$.
\end{abstract}

2000 Mathematics subject classification: primary 47A15, 46J15; secondary 47A20.

Keywords and phrases: invariant subspace, two variable Hardy space, wandering subspace.

\section{Introduction}

Let $\Gamma^{2}$ be the torus, that is, the Cartesian product of two unit circles $\Gamma$ in $\mathbb{C}$. Let $p=2$ or $p=\infty$. The usual Lebesgue spaces, with respect to the Haar measure $m$ on $\Gamma^{2}$, are denoted by $L^{p}=L^{p}\left(\Gamma^{2}\right)$, and $H^{p}=H^{p}\left(\Gamma^{2}\right)$ is the space of all $f$ in $L^{p}$ whose Fourier coefficients

$$
\hat{f}(j, \ell)=\int_{\Gamma^{2}} f(z, w) \bar{z}^{j} \bar{w}^{\ell} d m(z, w)
$$

vanish if at least one of $j$ and $\ell$ is negative. Then $H^{p}$ is called the Hardy space. As $\Gamma^{2}=\Gamma_{z} \times \Gamma_{w}, H^{p}\left(\Gamma_{z}\right)$ and $H^{p}\left(\Gamma_{w}\right)$ denote the one-variable Hardy spaces.

Let $P_{H^{2}}$ be the orthogonal projection from $L^{2}$ onto $H^{2}$. For $\phi$ in $L^{\infty}$, the Toeplitz operator $T_{\phi}$ is defined by

$$
T_{\phi} f=P_{H^{2}}(\phi f) \text { for all } f \in H^{2} .
$$

A closed subspace $M$ of $H^{2}$ is said to be forward-shift-invariant if $T_{z} M \subset M$ and $T_{w} M \subset M$, and a closed subspace $N$ of $H^{2}$ is said to be backward-shift-invariant if

This research was partially supported by Grant-in-Aid for Scientific Research, Japan Society for the Promotion of Science.

(c) 2008 Australian Mathematical Society 1446-7887/08 \$A2.00+0.00 
$T_{z}^{*} N \subset N$ and $T_{w}^{*} N \subset N$. Let $P_{M}$ and $P_{N}$ be the orthogonal projections from $H^{2}$ onto $M$ and $N$, respectively. In this paper, we assume that $M \oplus N=H^{2}$, that is, $P_{M}+P_{N}=I$ where $I$ is the identity operator on $H^{2}$. Let

$$
A=P_{M} T_{z} P_{N} \quad \text { and } \quad B=P_{N} T_{w}^{*} P_{M} .
$$

For $\phi$ in $H^{\infty}$,

$$
V_{\phi} f=P_{M}(\phi f) \quad \text { for all } f \in M \quad \text { and } \quad S_{\phi} f=P_{N}(\phi f) \quad \text { for all } f \in N .
$$

Suppose that

$$
\mathcal{V}=V_{z} V_{w}^{*}-V_{w}^{*} V_{z} \quad \text { and } \quad \mathcal{S}=S_{z} S_{w}^{*}-S_{w}^{*} S_{z}
$$

It is known [4] that $\left.A B\right|_{M}=\mathcal{V}$ and $\left.B A\right|_{N}=\mathcal{S}$. Guo and Yang [3] showed that $A B$ is Hilbert-Schmidt under some mild conditions. In this paper, we study $M$ or $N$ when $A$, $B, A B$ or $B A$ is of finite rank. Izuchi and Nakazi [4] described an invariant subspace $M$ or $N$ with $A=0$ or $B=0$. Mandrekar [6], Ghatage and Mandrekar [2] and Nakazi [7, 8] described an invariant subspace $M$ with $A B=0$. Izuchi and Nakazi [4] and Izuchi et al. [5] described an invariant subspace $N$ with $B A=0$.

For a forward-shift-invariant subspace $M$, put

$$
M_{1}=\operatorname{ker} V_{z}^{*}, \quad M_{2}=\operatorname{ker} V_{w}^{*} \quad \text { and } \quad M_{0}=M_{1} \cap M_{2} .
$$

These are called wandering subspaces for $M$. In this paper, [·] denotes the closed span. For a backward-shift-invariant subspace $N$, with $M=H^{2} \ominus N$, put

$$
N_{1}=\left[T_{z}^{*} M_{1}\right], \quad N_{2}=\left[T_{w}^{*} M_{2}\right] \quad \text { and } \quad N_{0}=N_{1} \cap N_{2} .
$$

These are called wandering subspaces for $N$.

In Section 2 we decompose and study $M$ and $N$ using the wandering subspaces $M_{1}, M_{2}, N_{1}$ and $N_{2}$. In Section 3 we study $M$ and $N$ when $A$ or $B$ is of finite rank. For an operator $K, r(K)$ denotes the rank of $K$. In Section 4 we show that $r(A B)=\operatorname{dim} N_{1} \cap N_{2}$ in general, and $r(B A)=\operatorname{dim} M_{1} \cap M_{2}$ under some mild conditions.

In this paper, for a bounded linear operator $X$ on $H^{2}, \operatorname{ran} X=X H^{2}$ and $\operatorname{ker} X=$ $\left\{f \in H^{2} \mid X f=0\right\}$.

\section{Wandering subspaces}

Let $M$ be a forward-shift-invariant subspace, and $N$ be a backward-shift-invariant subspace with $H^{2}=M \oplus N$. Put

$$
M_{z}^{\infty}=\bigcap_{n=1}^{\infty}\left\{f \in M \mid \bar{z}^{n} f \in M\right\} \quad \text { and } \quad M_{w}^{\infty}=\bigcap_{n=1}^{\infty}\left\{f \in M \mid \bar{w}^{n} f \in M\right\},
$$


and

$$
N_{z}^{\infty}=\bigcap_{n=1}^{\infty}\left\{f \in N \mid z^{n} f \in N\right\} \quad \text { and } \quad N_{w}^{\infty}=\bigcap_{n=1}^{\infty}\left\{f \in N \mid w^{n} f \in N\right\}
$$

In the case of one variable, $M_{z}^{\infty}=N_{z}^{\infty}=[0]$. In the case of two variables, $M_{z}^{\infty}$ is also always [0] but $N_{z}^{\infty}$ may not be [0]. In fact, if $N \supset q_{1} H^{2}\left(\Gamma_{z}\right)$ then $N_{z}^{\infty} \supset q_{1} H^{2}\left(\Gamma_{z}\right)$ where $q_{1}=q_{1}(z)$ is an inner function of one variable.

THEOREM 1. Let $N$ be a backward-shift-invariant subspace and $M=H^{2} \ominus N$.

(1) $M_{z}^{\infty}=M_{w}^{\infty}=[0]$ and $M=\sum_{n=0}^{\infty} \oplus T_{z}^{n} M_{1}=\sum_{n=0}^{\infty} \oplus T_{w}^{n} M_{2}$.

(2) $\quad N=\left[\bigcup_{n=0}^{\infty} T_{z}^{* n} N_{1}\right] \oplus N_{z}^{\infty}=\left[\bigcup_{n=0}^{\infty} T_{w}^{* n} N_{2}\right] \oplus N_{w}^{\infty}$.

PROOF. (1) is well known. To prove (2): if $f \in N_{z}^{\infty}$, then by definition $z^{n} f \in N$ for any $n \geq 1$, and hence $f$ is orthogonal to $\left[\bigcup_{n=0}^{\infty} T_{z}^{* n} N_{1}\right]$. Conversely, suppose that $f$ is orthogonal to $\bigcup_{n=0}^{\infty} T_{z}^{* n} N_{1}$. Since $f \perp N_{1}$, zf is orthogonal to $M_{1}+z M$ because $N_{1}=T_{z}^{*} M_{1}$ and $f \in N$. Hence $z f \in N$. Since $f \perp T_{z}^{*} N_{1}, z^{2} f$ is orthogonal to $M_{1}+z M$ because $T_{z}^{*} N_{1}=T_{z}^{* 2} M_{1}$ and $z f \in N$. Hence $z^{2} f \in N$. By repeating the same argument, we can show that $z^{n} f$ belongs to $N$ for any $n \geq 1$. This implies (2).

COROLlary 2. Let $N$ be a backward-shift-invariant subspace.

(1) $\quad N=N_{z}^{\infty}$ if and only if $N=H^{2}\left(\Gamma_{z}\right) \otimes\left(H^{2}\left(\Gamma_{w}\right) \ominus q_{2} H^{2}\left(\Gamma_{w}\right)\right)$ where $q_{2}=q_{2}(w)$ is an inner function of one variable.

(2) $\quad N=\left[\bigcup_{n=0}^{\infty} T_{z}^{* n} N_{1}\right]$ if and only if for each nonzero $f$ in $N$ there exists $n \geq 1$ such that $z^{n} f \notin N$.

Proof. (1) If $N=N_{z}^{\infty}$ then $N_{1}=0$ and so $T_{z}^{*} M_{1}=0$. Hence $M_{1} \subset H^{2}\left(\Gamma_{w}\right)$, so $M_{1}=q_{2} H^{2}\left(\Gamma_{w}\right)$ by a well-known theorem of Beurling [1]. Thus $M=q_{2} H^{2}$, and so

$$
N=H^{2}\left(\Gamma_{z}\right) \otimes\left(H^{2}\left(\Gamma_{w}\right) \ominus q_{2} H^{2}\left(\Gamma_{w}\right)\right) .
$$

Conversely, if $M=q_{2} H^{2}$ then $M_{1}=q_{2} H^{2}\left(\Gamma_{w}\right)$, and so $N_{1}=T_{z}^{*} M_{1}=0$. Part (2) is clear by (2) of Theorem 1.

By (1) of Theorem 1, both $M_{1}$ and $M_{2}$ are cyclic subspaces for $T_{z}$ and $T_{w}$ : that is,

$$
\left[\bigcup_{(n, m) \geq(0,0)} T_{z}^{n} T_{w}^{m} M_{j}\right]=M \quad \text { for } j=1,2 .
$$

It may happen that

$$
\left[\bigcup_{(n, m) \geq(0,0)} T_{z}^{n} T_{w}^{m} M_{0}\right]=M,
$$

where $M_{0}=M_{1} \cap M_{2}$. By (2) of Theorem 1 , if $N_{z}^{\infty}=[0]$ or $N_{w}^{\infty}=[0]$ then $N_{1}$ or $N_{2}$ is a cyclic subspace for $T_{z}^{*}$ and $T_{w}^{*}$ : that is, 


$$
\left[\bigcup_{(n, m) \geq(0,0)} T_{z}^{* n} T_{w}^{* m} N_{j}\right]=N \quad \text { for } j=1,2
$$

In general, $N_{0}$ may not be a cyclic subspace because $N_{0}=[0]$ may happen. We can ask whether $T_{z}^{*} M_{0}$ or $T_{w}^{*} M_{0}$ is a cyclic subspace for $T_{z}^{*}$ and $T_{w}^{*}$ because $N_{1} \supset T_{z}^{*} M_{0}$ and $N_{2} \supset T_{w}^{*} M_{0}$. However, this is not true. If $M=z H^{2}$ then $N=H^{2}\left(\Gamma_{w}\right)$ and $M_{0}=[z]$. Then $T_{w}^{*} M_{0}=[0]$ and $T_{z}^{*} M_{0}=[1]$.

EXAmple 1. Let $N=H^{2}\left(\Gamma_{z}\right)+H^{2}\left(\Gamma_{w}\right)$. Then the following hold.

(1) $N_{1}=w H^{2}\left(\Gamma_{w}\right), N_{2}=z H^{2}\left(\Gamma_{z}\right)$ and $N_{0}=[0]$.

(2) $\left[\bigcup_{n \geq 0} T_{z}^{* n} N_{1}\right]=w H^{2}\left(\Gamma_{w}\right), \quad\left[\bigcup_{n \geq 0} T_{w}^{* n} N_{2}\right]=z H^{2}\left(\Gamma_{z}\right) \quad$ and $\quad\left[\bigcup_{(n, m) \geq 0} T_{z}^{* n}\right.$ $\left.T_{w}^{* m} N_{0}\right]=[0]$.

(3) $N_{z}^{\infty}=H^{2}\left(\Gamma_{z}\right)$ and $N_{w}^{\infty}=H^{2}\left(\Gamma_{w}\right)$.

EXAmple 2. Let $N=\mathbb{C}$ and $M=z H^{2}+w H^{2}$. Then the following hold.

(1) $N_{1}=N_{2}=N_{0}=\mathbb{C}$.

(2) $\left[\bigcup_{n \geq 0} T_{z}^{* n} N_{1}\right]=\left[\bigcup_{n \geq 0} T_{w}^{* n} N_{2}\right]=\left[\bigcup_{(n, m) \geq(0,0)} T_{z}^{* n} T_{w}^{* m} N_{0}\right]=N$.

(3) $\quad N_{z}^{\infty}=N_{w}^{\infty}=[0]$.

EXAMPLE 3. Let

$$
N=\left(H^{2}\left(\Gamma_{z}\right) \ominus q_{1} H^{2}\left(\Gamma_{z}\right)\right) \otimes\left(H^{2}\left(\Gamma_{w}\right) \ominus q_{2} H^{2}\left(\Gamma_{w}\right)\right)
$$

and $M=q_{1} H^{2}+q_{2} H^{2}$, where $q_{1}=q_{1}(z)$ and $q_{2}=q_{2}(w)$ are inner functions of one variable.

(1) $\quad M_{1}=q_{1}\left(H^{2}\left(\Gamma_{w}\right) \ominus q_{2} H^{2}\left(\Gamma_{w}\right)\right) \oplus q_{2} H^{2}\left(\Gamma_{w}\right)$ and $M_{2}=q_{2}\left(H^{2}\left(\Gamma_{z}\right) \ominus q_{1} H^{2}\right.$ $\left.\left(\Gamma_{z}\right)\right) \oplus q_{1} H^{2}\left(\Gamma_{z}\right)$.

(2) $\quad N_{1}=\left(T_{z}^{*} q_{1}\right)\left(H^{2}\left(\Gamma_{w}\right) \ominus q_{2} H^{2}\left(\Gamma_{w}\right)\right), N_{2}=\left(T_{w}^{*} q_{2}\right)\left(H^{2}\left(\Gamma_{z}\right) \ominus q_{1} H^{2}\left(\Gamma_{z}\right)\right)$ and $N_{0}=\left\langle\left(T_{z}^{*} q_{1}\right)\left(T_{w}^{*} q_{2}\right)\right\rangle$.

(3) $\left[\bigcup_{n \geq 0} T_{z}^{* n} N_{1}\right]=\left[\bigcup_{n \geq 0} T_{w}^{* n} N_{2}\right]=\left[\bigcup_{(n, m) \geq(0,0)} T_{z}^{* n} T_{w}^{* m} N_{0}\right]=N$.

PROOF. (2) and (3) follow from (1). It is known [4] that

$$
\begin{aligned}
M= & q_{2} H^{2} \oplus q_{1}\left(H^{2} \ominus q_{2} H^{2}\right)=\left(H^{2}\left(\Gamma_{z}\right) \otimes q_{2} H^{2}\left(\Gamma_{w}\right)\right) \\
& \oplus\left\{q_{1} H^{2}\left(\Gamma_{z}\right) \otimes\left(H^{2}\left(\Gamma_{w}\right) \ominus q_{2} H^{2}\left(\Gamma_{w}\right)\right)\right\} .
\end{aligned}
$$

Hence (1) follows.

$$
\text { 3. } r(A)<\infty \text { or } r(B)<\infty
$$

Recall that $A=P_{M} T_{z} P_{N}$ and $B=P_{N} T_{w}^{*} P_{M}$ (see Introduction). In this section, we are interested in the case when $A$ or $B$ is of finite rank. We know a characterization 
of $A=0$ or $B=0$ (see [3]). In fact, $A=0$ if and only if $N=H^{2}$ or $N=H^{2} \ominus q H^{2}$ where $q=q(w)$ is an inner function of one variable, and $B=0$ if and only if $M=[0]$ or $M=q H^{2}$ where $q=q(z)$ is an inner function of one variable. In one-variable Hardy space, either $A$ is of rank one for any $N$ or $B$ is of rank one for any $M$.

LEMMA 3. Let $M$ be a forward-shift-invariant subspace of $H^{2}$ and $N=H^{2} \ominus M$.

(1) $[\operatorname{ran} A] \subseteq M_{1}$ and $\operatorname{ker} A=\left\{f \in N \mid T_{z} f \in N\right\} \oplus M$.

(2) $\left[\operatorname{ran} A^{*}\right]=N_{1}$ and $\operatorname{ker} A^{*}=\left\{f \in M \mid T_{z}^{*} f \in M\right\} \oplus N$.

(3) $\quad M_{1}=[\operatorname{ran} A] \oplus\left\{\operatorname{ker} A^{*} \ominus\left(T_{z} M \oplus N\right)\right\}$.

(4) $\quad M=[\operatorname{ran} A] \oplus\left(\operatorname{ker} A^{*} \ominus N\right)$ and $N=\left[\operatorname{ran} A^{*}\right] \oplus(\operatorname{ker} A \ominus M)$.

Proof. (1) By the definitions, $[\operatorname{ran} A]=\left[P_{M} T_{z} N\right] \subseteq M_{1}$ because $T_{z} N$ is orthogonal to $T_{z} M$ and

$$
\text { ker } A=\left\{f \in N \mid T_{z} f \in N\right\} \oplus M \text {. }
$$

(2) Since $T_{z}^{*} M=T_{z}^{*} M_{1} \oplus M,\left[\operatorname{ran} A^{*}\right]=\left[T_{z}^{*} M_{1}\right]=N_{1}$. By definition,

$$
\operatorname{ker} A^{*}=\left\{f \in M \mid T_{z}^{*} f \in M\right\} \oplus N
$$

(3) is clear from (1) and the fact that $H^{2}=[\operatorname{ran} A] \oplus \operatorname{ker} A^{*}$. (4) is clear from (1), (2) and the fact that $H^{2}=\left[\operatorname{ran} A^{*}\right] \oplus \operatorname{ker} A$.

LEMMA 4. Let $M$ be a forward-shift-invariant subspace of $H^{2}$ and $N=H^{2} \ominus M$.

(1) $[\operatorname{ran} A]=M_{1} \ominus\left(M_{1} \cap \operatorname{ker} T_{z}^{*}\right)$.

(2) $\quad \operatorname{ker} A^{*}=\left(M_{1} \cap \operatorname{ker} T_{z}^{*}\right) \oplus T_{z} M \oplus N$.

ProOf. (1) Since $T_{z} N \perp \operatorname{ker} T_{z}^{*}, \quad T_{z} N \perp M_{1} \cap \operatorname{ker} T_{z}^{*}$, so $P_{M} T_{z} N \perp M_{1} \cap \operatorname{ker} T_{z}^{*}$. Hence, by (1) of Lemma 3, $[\operatorname{ran} A] \subseteq M_{1} \ominus\left(M_{1} \cap \operatorname{ker} T_{z}^{*}\right)$. If $f \in M_{1}$ and $f \perp \operatorname{ran} A$, then $f \perp T_{z} N$ and so $T_{z}^{*} f \perp N$. Hence $T_{z}^{*} f \in N \cap M$, because $T_{z}^{*} M_{1} \perp M$. Hence $T_{z}^{*} f=0$. (2) follows from (1), by (2) of Lemma 3 .

LEMMA 5. Let $M$ be a forward-shift-invariant subspace of $H^{2}$. If $[\operatorname{ran} A] \neq M_{1}$, then $M_{1}=[\operatorname{ran} A] \oplus q_{2} H^{2}\left(\Gamma_{w}\right)$.

Proof. By Lemma 4, $M_{1} \ominus[\operatorname{ran} A]=M_{1} \cap \operatorname{ker} T_{z}^{*}$ and $M_{1} \cap \operatorname{ker} T_{z}^{*} \subset H^{2}\left(\Gamma_{w}\right)$ because $\operatorname{ker} T_{z}^{*}=H^{2}\left(\Gamma_{w}\right)$. Hence $w\left(M_{1} \cap \operatorname{ker} T_{z}^{*}\right) \perp z M$, and so

$$
w\left(M_{1} \cap \operatorname{ker} T_{z}^{*}\right) \subseteq M_{1} \cap \operatorname{ker} T_{z}^{*}
$$

By a theorem of Beurling [1], $M_{1} \ominus[\operatorname{ran} A]=q_{2} H^{2}\left(\Gamma_{w}\right)$ for some one-variable inner function $q_{2}=q_{2}(w)$. 
THEOREM 6. Let $M$ be a nonzero forward-shift-invariant subspace.

(1) If $r(A)<\infty$, then there is a one-variable inner function $q_{2}=q_{2}(w)$ such that

$$
M_{1}=\operatorname{ran} A \oplus q_{2} H^{2}\left(\Gamma_{w}\right) \quad \text { and } \quad M=q_{2} H^{2} \oplus\left\{\sum_{j=0}^{\infty} \oplus(\operatorname{ran} A) z^{j}\right\}
$$

where $q_{2}=q_{2}(w)$ is a one-variable inner function.

(2) If $r(B)<\infty$, then there is a one-variable inner function $q_{1}=q_{1}(z)$ such that

$$
M_{2}=\operatorname{ran} B^{*} \oplus q_{1} H^{2}\left(\Gamma_{z}\right) \quad \text { and } \quad M=q_{1} H^{2} \oplus\left\{\sum_{j=0}^{\infty} \oplus\left(\operatorname{ran} B^{*}\right) w^{j}\right\}
$$

where $q_{1}=q_{1}(z)$ is a one-variable inner function.

(3) If $r(A)<\infty$ and $r(B)<\infty$ then there exist two inner functions $q_{1}=q_{1}(z)$ and $q_{2}=q_{2}(w)$ such that $q_{1} H^{2}+q_{2} H^{2}$ is a closed forward-shift-invariant subspace, $M \supseteq q_{1} H^{2}+q_{2} H^{2}$, and

$$
\operatorname{dim}\left\{M_{1}+M_{2}\right\} /\left\{q_{1} H^{2}\left(\Gamma_{z}\right)+q_{2} H^{2}\left(\Gamma_{w}\right)\right\} \leq r(A)+r(B) .
$$

Proof. Since $\operatorname{dim} M_{1}=\infty$ by [7, Theorem 3], if $r(A)<\infty$ then $[\operatorname{ran} A] \neq M_{1}$, and so by Lemma 3, $M_{1}=[\operatorname{ran} A] \oplus q_{2} H^{2}\left(\Gamma_{w}\right)$ for some one-variable inner function $q_{2}=q_{2}(w)$. This implies (1). If $r(B)<\infty$, then $r\left(B^{*}\right)<\infty$. Since $B^{*}=P_{M} T_{w} P_{N}$, (1) implies (2). If $r(A)<\infty$ and $r(B)<\infty$, (1) and (2) imply (3) because it is known [4] that $q_{1} H^{2}+q_{2} H^{2}$ is closed.

\section{COROLlary 7.}

(1) If $A=0$, then $M=[0]$ or $M=q_{2} H^{2}$ for some one-variable inner function $q_{2}=q_{2}(w)$.

(2) If $B=0$, then $M=[0]$ or $M=q_{1} H^{2}$ for some one-variable inner function $q_{1}=q_{1}(z)$.

\section{COROLlary 8.}

(1) If $0 \leq n \leq \infty$ and $0 \leq m \leq \infty$, then there exist invariant subspaces $M$ and $N$ such that $r(A)=n$ and $r(B)=m$.

(2) If $r(B)=0$, then $r(A)=0$ or $r(A)=\infty$. If $r(A)=0$, then $r(B)$ is 0 or $\infty$.

Proof. (1) Let $1 \leq n<\infty$ and $1 \leq m<\infty$. Suppose that $M=z^{m} H^{2}+w^{n} H^{2}$. Then

$$
\begin{aligned}
& M_{1}=w^{n} H^{2}\left(\Gamma_{w}\right)+\left[1, w, \ldots, w^{n-1}\right] z^{m} \quad \text { and } \\
& M_{2}=z^{m} H^{2}\left(\Gamma_{z}\right)+\left[1, z, \ldots, z^{m-1}\right] w^{n} .
\end{aligned}
$$

By (1) and (2) of Theorem 6, $r(A)=n$ and $r(B)=m$.

(2) If $r(B)=0$, then by (2) of Corollary 7, $M=[0]$ or $M=q_{1} H^{2}$ where $q_{1}=q_{1}(z)$ is a one-variable inner function. If $M=[0]$ then $r(A)=0$ by definition. If $M=q_{1} H^{2}$ 
then $M_{1}=q_{1} H^{2}\left(\Gamma_{w}\right)$, and so if $r(A)<\infty$ then by (1) of Theorem $6 M_{1} \supset q_{2} H^{2}\left(\Gamma_{w}\right)$ for some one-variable inner function $q_{2}=q_{2}(w)$. This implies that $q_{1}$ is constant. Hence $M=H^{2}$, and so $A=0$.

For a finite Blaschke product $q, \operatorname{deg} q$ denotes the number of zeros of $q$ counting multiplicity.

COROLlary 9. If $M=q_{1} H^{2}+q_{2} H^{2}$ where $q_{1}=q_{1}(z)$ and $q_{2}=q_{2}(w)$ are onevariable inner functions, then

$$
[\operatorname{ran} A]=q_{1}\left(H^{2}\left(\Gamma_{w}\right) \ominus q_{2} H^{2}\left(\Gamma_{w}\right)\right) \quad \text { and }\left[\operatorname{ran} B^{*}\right]=q_{2}\left(H^{2}\left(\Gamma_{z}\right) \ominus q_{1} H^{2}\left(\Gamma_{z}\right)\right) \text {. }
$$

If $r(A)<\infty$ and $r(B)<\infty$, then $r(A)=\operatorname{deg} q_{2}$ and $r(B)=\operatorname{deg} q_{1}$.

Corollary 10. Let $M$ be a forward-shift-invariant subspace. If $M$ is of finite codimension $n$, then $r(A) \leq n, r(B) \leq n$, and $M \supseteq q_{1} H^{2}+q_{2} H^{2}$ where $q_{1}=q_{1}(z)$ and $q_{2}=q_{2}(w)$ are one-variable finite Blaschke products.

Proof. By the definitions of $A$ and $B$, it is clear that $r(A) \leq n$ and $r(B) \leq n$. The second statement follows from (3) of Theorem 6.

Proposition 11. Let $M$ be a forward-shift-invariant subspace. Then $M \supseteq q_{1} H^{2}$ $+q_{2} H^{2}$ for some one-variable inner functions $q_{1}=q_{1}(z)$ and $q_{2}=q_{2}(w)$ if and only if $[\operatorname{ran} A] \neq M_{1}$ and $\left[\operatorname{ran} B^{*}\right] \neq M_{2}$.

PROOF. The 'if' part is clear by Lemma 5. If $M \supseteq q_{1} H^{2}$, then $q_{1} H^{2}\left(\Gamma_{z}\right)$ is orthogonal to $w M$, and so $q_{1} H^{2}\left(\Gamma_{z}\right) \subseteq M_{2}$. Hence Lemma 4 implies that $\left[\operatorname{ran} B^{*}\right] \neq M_{2}$. Similarly we can prove that if $M \supseteq q_{2} H^{2}$ then $[\operatorname{ran} A] \neq M_{1}$.

Proposition 12. $N_{1}=\left[\operatorname{ran} A^{*}\right]$ and $N_{2}=[\operatorname{ran} B]$. Hence $\operatorname{dim} N_{1}=r(A)$ and $\operatorname{dim} N_{2}=r(B)$.

PROOF. This follows from (2) of Lemma 3.

\section{4. $\boldsymbol{r}(\boldsymbol{A B})<\infty$ or $\boldsymbol{r}(\boldsymbol{B} A)<\infty$}

Let $M$ be a forward-shift-invariant subspace and $N=H^{2} \ominus M$. Recall the definitions of $\mathcal{V}$ and $\mathcal{S}$ in the Introduction. It is known [4] that $\left.A B\right|_{M}=\mathcal{V}$ and $\left.B A\right|_{N}=\mathcal{S}$. Then $A B=0$ if and only if $\mathcal{V}=0$, and $B A=0$ if and only if $\mathcal{S}=0$. We know the characterization of an invariant subspace such that $A B=0$ or $B A=0$. In fact, it is known (see [6-8]) that $A B=0$ if and only if $M=q H^{2}$ for some inner function $q$. Recently it was proved (see $[4,5]$ ) that $B A=0$ if and only if

$$
\begin{aligned}
& N=\left(H^{2}\left(\Gamma_{z}\right) \ominus q_{1} H^{2}\left(\Gamma_{z}\right)\right) \otimes\left(H^{2}\left(\Gamma_{w}\right) \ominus q_{2} H^{2}\left(\Gamma_{w}\right)\right), \\
& N=\left(H^{2}\left(\Gamma_{z}\right) \ominus q_{1} H^{2}\left(\Gamma_{z}\right)\right) \otimes H^{2}\left(\Gamma_{w}\right), \quad \text { or } \\
& N=H^{2}\left(\Gamma_{z}\right) \otimes\left(H^{2}\left(\Gamma_{w}\right) \ominus q_{2} H^{2}\left(\Gamma_{w}\right)\right),
\end{aligned}
$$

where $q_{1}=q_{1}(z)$ and $q_{2}=q_{2}(w)$ are one-variable inner functions. In this section, we study invariant subspaces such that $r(A B)<\infty$ or $r(B A)<\infty$. 
LEMMA 13. Let $M$ be a forward-shift-invariant subspace and $N=H^{2} \ominus M$.

(1) $\quad r(B A)=\operatorname{dim}\left(\left[P_{M} T_{z} N\right] \cap\left[P_{M} T_{w} N\right]\right)$.

(2) $\quad r(A B)=\operatorname{dim}\left(\left[P_{N} T_{z}^{*} M\right] \cap\left[P_{N} T_{w}^{*} M\right]\right)$.

Proof. (1) Since $\left[B A H^{2}\right]=[B[\operatorname{ran} A]], r(B A)=\operatorname{dim}\left((\operatorname{ker} B)^{\perp} \cap[\operatorname{ran} A]\right)$. This implies (1) because $(\operatorname{ker} B)^{\perp}=\left[\operatorname{ran} B^{*}\right]=\left[P_{M} T_{w} N\right]$ and $[\operatorname{ran} A]=\left[P_{M} T_{z} N\right]_{2}$. (2) can be proved similarly.

THEOREM 14. Let $M$ be a forward-shift-invariant subspace of $H^{2}$ and $N=H^{2} \ominus M$.

(1) If $M_{1} \cap \operatorname{ker} T_{z}^{*}=[0]$ and $M_{2} \cap \operatorname{ker} T_{w}^{*}=[0]$, then $r(B A)=\operatorname{dim} M_{1} \cap M_{2}$.

(2) $r(A B)=\operatorname{dim} N_{1} \cap N_{2}$.

Proof. (1) By (1) and (2) of Lemma 3,

$$
[\operatorname{ran} A]=\left[P_{M} T_{z} N\right] \subseteq M_{1} \text { and }\left[\operatorname{ran} B^{*}\right]=\left[P_{M} T_{w} N\right] \subseteq M_{2} .
$$

By Lemma 4, if $M_{1} \cap \operatorname{ker} T_{z}^{*}=[0]$ then $\left[P_{M} T_{z} N\right]=M_{1}$, and if $M_{2} \cap \operatorname{ker} T_{w}^{*}=[0]$ then $\left[P_{M} T_{w} N\right]=M_{2}$. Hence, $r(B A)=\operatorname{dim} M_{1} \cap M_{2}$ by Lemma 13 .

(2) Since $\left[P_{N} T_{z}^{*} M\right]=\left[P_{N} T_{z}^{*} M_{1}\right]=N_{1}$ and $\left[P_{N} T_{w}^{*} M\right]=\left[P_{N} T_{w}^{*} M_{2}\right]=N_{2}$, by Lemma $13 r(A B)=\operatorname{dim} N_{1} \cap N_{2}$.

In (1) of Theorem 14, we need the condition $M_{1} \cap \operatorname{ker} T_{z}^{*}=M_{2} \cap \operatorname{ker} T_{w}^{*}=[0]$. In fact, $M_{1} \cap M_{2}$ is always nontrivial but $B A$ may be zero.

\section{References}

[1] A. Beurling, 'On two problems concerning linear transformations in Hilbert space', Acta Math. 81 (1949), 239-255.

[2] P. Ghatage and V. Mandrekar, 'On Beurling type invariant subspaces of $L^{2}\left(\mathbf{T}^{2}\right)$ and their equivalence', J. Operator Theory 20 (1988), 31-38.

[3] K. Guo and R. Yang, 'The core function of submodules over the bidisk', Indiana Univ. Math. J. 53 (2004), 205-222.

[4] K. Izuchi and T. Nakazi, 'Backward shift invariant subspaces in the bidisc', Hokkaido Math. J. 3 (2004), 247-254.

[5] K. Izuchi, T. Nakazi and M. Seto, 'Backward shift invariant subspaces in the bidisc II', J. Operator Theory 51 (2004), 361-376.

[6] V. Mandrekar, 'The validity of Beurling theorems in polydiscs', Proc. Amer. Math. Soc. 103 (1988), $145-148$.

[7] T. Nakazi, 'Certain invariant subspaces of $H^{2}$ and $L^{2}$ on a bidisc', Canad. J. Math. 40 (1988), 1272-1280.

[8] _ 'Invariant subspaces in the bidisc and commutators', J. Aust. Math. Soc. 56 (1994), 232242.

[9] $\longrightarrow$, 'Homogeneous polynomials and invariant subspaces in the polydiscs', Arch. Math. 58 (1992), 56-63.

TAKAHIKO NAKAZI, Department of Mathematics, Faculty of Science,

Hokkaido University, Sapporo 060-0810, Japan

e-mail: nakazi@math.sci.hokudai.ac.jp 